\title{
Cultural identity, clothing and common mental disorder: a prospective school-based study of white British and Bangladeshi adolescents
}

\author{
K Bhui, ${ }^{1}$ Y Khatib, ${ }^{1}$ R Viner, ${ }^{2}$ E Klineberg, ${ }^{1}$ C Clark, ${ }^{1}$ J Head, ${ }^{1,2}$ S Stansfeld ${ }^{1}$
}

${ }^{1}$ Centre for Psychiatry, Barts \& The London, Queen Mary's School of Medicine and Dentistry, London, UK; ${ }^{2}$ University College London, London, UK

Correspondence to: Professor K Bhui, Centre for Psychiatry, Barts \& The London Medical School, Queen Mary's University of London, London E1 4NS, UK; k.s.bhui@qmul.ac.uk

Accepted 1 August 2007

\section{ABSTRACT}

Objective: Cultural integration is the healthiest outcome for young people living in multicultural societies. This paper investigates the influence of different cultural identities on the risk of common mental disorders among Bangladeshi and white British pupils.

Design: The cultural identity of 11-14-year-old school pupils was assessed by their preferences for friends and clothes of their own or other cultural groups; using this information pupils were classified into traditional, integrated, assimilated or marginalised groups. We undertook prospective analyses of cultural identity and its impacts on the later mental health of young people.

Setting: East London.

Participants: In 2001, white British (573) and Bangladeshi (682) school pupils from a representative sample of schools completed a self-report questionnaire that assessed their cultural, social and health characteristics. In 2003, 383 white British and 517 Bangladeshi pupils were resurveyed and completed measures of mental health.

Main outcome measure: Strengths and difficulties questionnaire.

Results: Bangladeshi pupils preferring clothes from their own cultural group (traditional clothing) were less likely to have later mental health problems when compared with Bangladeshi pupils showing an equal preference for clothing from their own and other cultures (integrated clothing; odds ratio (OR) $0.3,95 \% \mathrm{Cl} 0.1$ to 0.9 ). In gender-specific analyses, this finding was sustained only among Bangladeshi girls (OR 0.1, 95\% Cl 0.1 to 0.7). Integrated clothing choices were least risky only for white British adolescents. Friendship choices showed no prospective associations with later mental health problems.

Conclusions: Cultural identity, expressed by clothing preferences, influences mental health; the effects differ by gender and ethnic group.

Adolescence is a vulnerable period for the development of mental health problems. ${ }^{1-3}$ Although previous work demonstrates ethnic variations of the incidence and prevalence of mental health problems, ${ }^{4}$ there is uncertainty about which specific lifestyle and behavioural correlates of ethnicity confer health risks or advantages. Discrimination, place of birth, gender roles, religious practice and migration stresses may all be relevant. Little attention has been given to young people's cultural identity and the health risks associated with adaptation to living in multiethnic urban areas. $^{4-6}$ The magnitude of health risks and mediating mechanisms are important to understand in order to inform health policy, as well as immigration, social and education policies. ${ }^{78}$ For example, the individual freedom to wear some forms of traditional Muslim clothing and religious symbols in public places such as schools, law courts and airlines has recently been challenged. ${ }^{9}$ In some countries such as France, these individual freedoms are not permitted as they are perceived to erode social and community integration. ${ }^{10}$ Like other expressions of personal and ethnic group identity, clothing can influence group perceptions of superiority, inferiority, worth and value, and therefore can impact on self-esteem and wellbeing when social and cultural norms are being negotiated. ${ }^{11-13}$ The recent interest in clothing as an external marker of traditional religious identity among Muslims in Europe has informed debates about the value of multiculturalism and the optimal immigration and social policies for the integration of migrants.

Acculturation arises as a consequence of groups with different cultures coming into continuous first hand contact leading to changes in the original cultural patterns of either or both groups. ${ }^{14}{ }^{15}$ The pressure to change lifestyle, attitudes and behaviours may lead to emotional distress, ${ }^{45}$ and minority status itself may lead to a higher risk of illness. ${ }^{16} 17$ There are several methodological challenges in research on this subject. First, there are different conventions about choosing ethnic group, race, or culture as a category. When referring to the literature we use terms used in original studies, and rely on cultural identity as a global term that reflects racial and ethnic identity issues. Previous studies of acculturation and mental health among young people have been of a cross-sectional design and can not unravel the direction of associations between cultural identity and mental health. ${ }^{16}{ }^{18-21}$ Existing methods to measure cultural identity enquire about attitudes, behaviours and cultural preferences for friends, clothes, diet, language of films, news material and education and marital partners. ${ }^{5-7} 22$ Friendship and clothing choices are especially important markers of cultural identity during adolescence. ${ }^{1323-25}$ Furthermore, boys and girls differ on levels of intimacy in friendships and both select friends of the same gender and race, ${ }^{2-27}$ perhaps as cross-cultural friendships are less reciprocal. ${ }^{24}{ }^{26}$ Friends are an important source of social support, ${ }^{28}$ but the pattern of selecting friends from the same or other cultural groups may differ according to the social venue. For example, in one study, most children had school friends from racial groups other than their own, but only $28 \%$ saw 
them outside of school. ${ }^{29}$ Clothing is also an important form of expression for identity during adolescents' transition from child to adult roles. Although less is known about conformity to culturally sanctioned clothing choices as a determinant of mental health problems, gender differences do exist: girls choose clothes for affective and emotional benefits, whereas boys choose clothing to achieve their ideal image of themselves. ${ }^{30} 31$ The cultural influences on these choices require further investigation.

The model of acculturation used in this study classifies individuals into traditional, marginalised, integrated and assimilated identity groups..$^{5-7}$ Using this model, a previous study demonstrated that adolescents with integrated friendship choices (equally likely to have friends of other and own cultural group) were $43 \%$ less likely to have a common (non-psychotic) mental health problem when compared with adolescents marginalised from their own and other cultural groups. ${ }^{67}$ Girls with integrated clothing choices and boys with integrated friendship choices had a lower risk of mental health problems compared with their marginalised counterparts. This paper presents the findings from the first longitudinal study on cultural identity expressed through clothing and friendship choices.

\section{HYPOTHESES}

The existing literature and our previous work helped formulate our a priori hypotheses. We hypothesised that:

- Integrated cultural identities on the basis of friendship and on the basis of clothing choices (equal likelihood of having friends or wearing clothes from own and other cultures) were predictive of lowest levels of later mental health problems among adolescents of both Bangladeshi and white British origin.

- Traditional identities on the basis of friendship and clothing choices (a preference for having friends or wearing clothing from ones own cultural group) were associated with better mental health only among Bangladeshi pupils.

- We hypothesised that there may be school-level effects; that for any one ethnic group, the schools with a greater proportion of young people of that group with traditional cultural identity show a lower individual risk of mental health problems among pupils from that ethnic group.

\section{METHODS \\ Sample}

The Research with East London Adolescents Community Health Survey (RELACHS) is a longitudinal school-based survey of a representative sample of young people in east London. ${ }^{32}$ The schools were located in the London boroughs of Hackney, Tower Hamlets and Newham. These have the most ethnic and cultural diversity in the country and have some of the largest refugee populations in the United Kingdom.

In 2001, 30 of the 43 secondary schools in east London were randomly selected and balanced to ensure representation of single and mixed sex schools. In the 28 schools that agreed to participate in phase 1 (2001), two representative mixed ability classes were selected from each of year 7 (aged 11-12 years) and year 9 (aged 13-14 years). In phase 1, 573 white British and 682 Bangladeshi adolescents completed the survey. This paper focuses only on Bangladeshi ( $\mathrm{N}=517 ; 75.8 \%$ follow-up) and white British (383; 66.8\% follow-up) adolescents who completed the mental health outcome measure in phase 2 , having completed these measures in phase 1 . Parents and pupils were informed by letter of the study. Written informed consent was sought from each school's head teacher, and from each participating adolescent; parents were fully informed about the study and given the opportunity to opt out. In addition, we met with local teachers, parents, health and social care professionals, to seek advice on the research process. The local research ethics committee approved both phases of the study.

\section{Mental health measures}

Mental health was measured using the self-report strengths and difficulties questionnaire (SDQ) completed in classrooms at the second phase of data collection. ${ }^{33}{ }^{34}$ The SDO is a brief behavioural screening questionnaire for 3-16 year olds. ${ }^{34}{ }^{34}$ It functions as well as the Rutter questionnaires ${ }^{35}$ and was demonstrated to be psychometrically valid in large surveys of young people in multicultural community samples, including studies among Bangladeshi young people..$^{35-37}$

A total difficulties score $(0-40)$ is derived by summing four subscale scores (emotional symptoms, conduct problems, peer problems, hyperactivity). Young people were considered to have "mental health problems" if they scored 17.5 or more, based on scores in national data in which approximately $10 \%$ (9.4\% boys, $9.0 \%$ girls) of the sample scored within the "high scorer" band. ${ }^{35}$ This threshold on self-report questionnaires distinguished those receiving mental care from those not receiving it. ${ }^{34}{ }^{35}$ The selfreport versions of this instrument show acceptable levels of correlation with parent and teacher ratings. ${ }^{34}$

\section{Ethnicity and culture indicators}

Ethnic group ratings were self-reported, and based on the 2001 England and Wales census; we also asked questions on place of birth, language use and religious group. These categories reflect the nature of ethnic groups in the United Kingdom, and are accepted as cogent research categories in the census and population research. In this health survey we included questions on cultural preferences for friends and clothes to assess cultural identity. ${ }^{6738} 39$ The methods used to classify cultural identity have previously been published. ${ }^{67}$ The strength of affiliation to other or own cultural group was rated by scores to four questions on a four-item Likert scale. Two questions asked about preferences for clothes and then a further two questions asked about preferences for friends. The first question asks about choosing clothing and friendships from the respondent's "own" cultural group. The second asks about choosing friends and clothing from "other cultures" (see Berry ${ }^{5}$ and Bhui and colleagues $^{67}$ for methods to classify cultural identity). The responses to these questions for any one domain (friendship or clothing) can be combined to classify pupils into a traditional group (choose only their own cultural group), an assimilated group (choose only from other cultural groups), an integrated group (choose from both cultural groups) and a marginalised group (choose neither from their own nor other cultural groups). Previous work included piloting and debriefing to improve face and content validity and to ensure optimal response rates. ${ }^{67}$

\section{Analyses}

All analyses were weighted for the proportion of non-responders per cluster (using $p w$ in Stata; Stata Corp, College Station, Texas, USA); standard errors were adjusted for cluster sampling using cluster and regression models used survey estimation (svy) commands in Stata 5.0. Potential confounding factors measured at the first phase were year group, school, borough, eligibility for free school meals (a valid proxy measure of household 
Table 1 Descriptive data: demographic and sociocultural characteristics by strengths and difficulties questionnaire case status at second phase

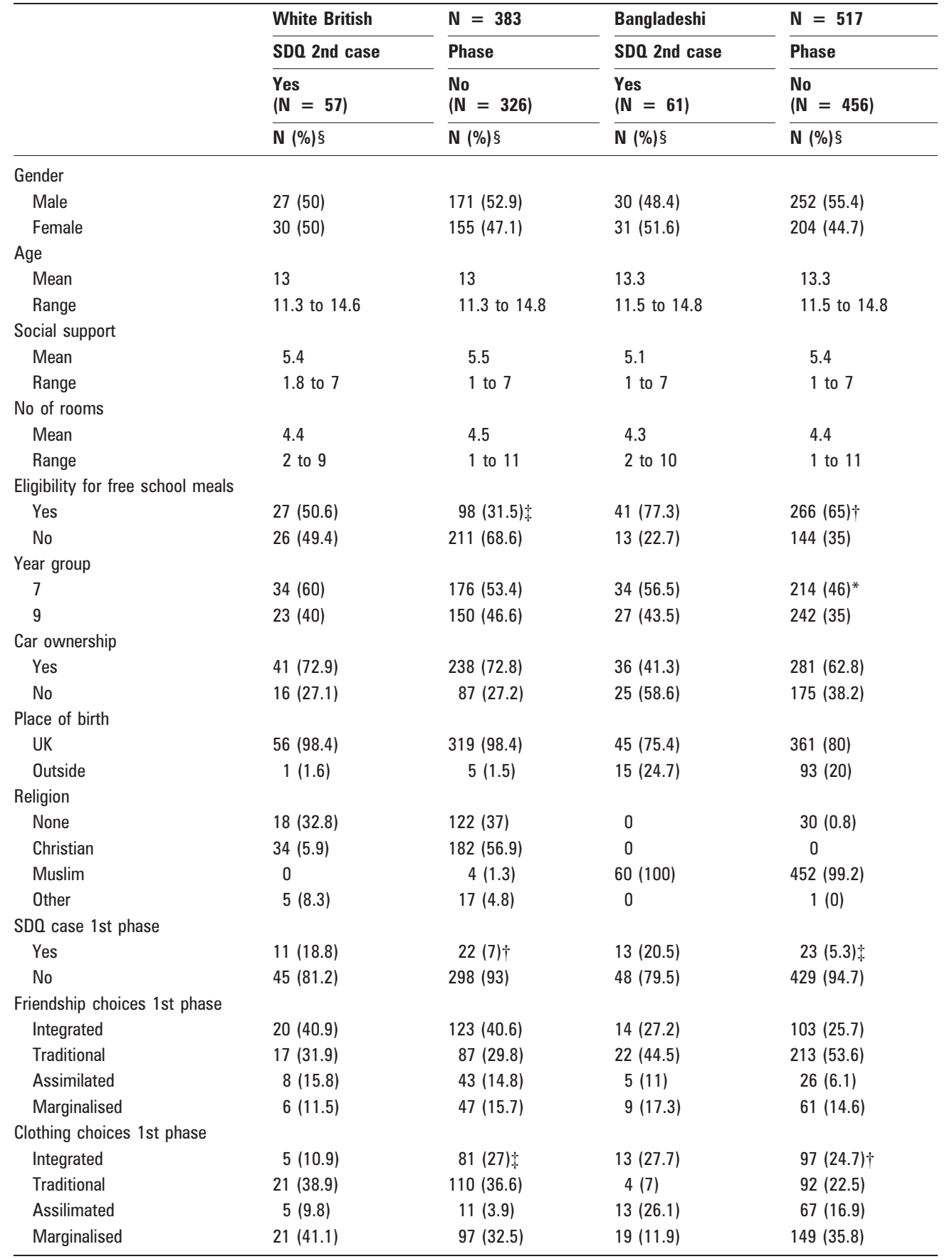

SDO, Strengths and difficulties questionnaire.

${ }^{*} \mathrm{p}<0.1, \uparrow \mathrm{p}<0.05, \pm \mathrm{p}<0.01$.

$\S$ Percentages are for number of cases/total number of subjects with complete data for each variable, for each ethnic group separately.

income), parental car ownership (none or one or more as a measure of socioeconomic status) and number of rooms in the home (as a measure of affluence). The "multidimensional scale of perceived social support" consists of 12 items, each scored on a Likert scale from 1 (disagree very strongly) to 7 (agree very strongly). Summation of the 12 -item scores provides a total score for overall social support. ${ }^{40}$ The friends subscale of the "multidimensional scale of perceived social support" assessed friendship support as a potential confounder. ${ }^{67}$ We adjusted for social support in regression models in which social support may confound the relationship between the friendship-based classification of identity and mental health problems. We also adjusted for mental health problems at baseline (SDQ case or not). Potential explanatory factors included gender and ethnic group.

\section{Individual level effects}

Univariate associations between first phase characteristics and mental health problems at the second phase were assessed (table 1). Associations between cultural identity at baseline and mental health at follow-up were investigated in unadjusted and adjusted logistic regression models for each ethnic group 
separately. Adjustment was made for all potential confounding factors and age and gender. We then stratified these models to assess gender-specific effects. In logistic regression models the integrated group was the reference group as it has previously been reported to be the healthiest group with the lowest risk. ${ }^{5} \mathrm{~A}$ gender by year group interaction was included in non-genderstratified model as girls were more likely to be in year 9 than 7 (odds ratios (OR) 1.57, 95\% confidence interval (CI) 1.01 to $2.42, \mathrm{p}=0.04)$.

\section{School-level effects}

School-level effects were assessed by including a school-level variable: the proportion of the school with traditional clothing identity for any one ethnic group. There were separate schoollevel variables for white British and Bangladeshi pupils and separate models were developed for each of these ethnic groups. Multilevel analyses were undertaken using MLWin software and included age, gender, car ownership, eligibility for free meals at school, place of birth and the SDQ score at phase 1; the measure of mental health was a binary measure applying the same threshold as for individual-level analyses to the SDQ score at phase 2 . These analyses were not weighted, so significant findings reflect valid relationships between the variables of interest, rather than precise estimates of the magnitude of the relationship found in population samples.

\section{RESULTS}

\section{Mental health problems, cultural identity and ethnic group}

Demographic characteristics are set out in table 1. In prospective analyses there were no significant associations between friendship-based measures of cultural identity and mental health outcomes, for Bangladeshi or white British adolescents overall or for gender-specific analyses (tables 2 and 3). More detailed modelling and multilevel analyses were not undertaken for friendship-based measures of cultural identity.

Traditional clothing choices among Bangladeshis were associated with a lower risk of mental health problems in unadjusted and adjusted analyses (table 2). White British adolescents classified as having integrated clothing choices were at lowest risk of mental health problems compared with those classified as having traditional, marginalised or assimilated cultural identities. More detailed modelling and multilevel analyses were thus undertaken for clothing-based measures of cultural identity.

\section{Effects of gender by ethnic group}

The impact of clothing-based measures of identity was examined by gender (table 3). Bangladeshi girls with traditional clothing choices were less likely to have mental health problems at follow-up. In contrast, white British girls making integrated clothing choices had the lowest risk of mental health problems at follow-up.

Compared with their counterparts who made integrated clothing choices, Bangladeshi boys with assimilated clothing choices had a lower risk of mental health problems at follow-up. In contrast, white British boys with integrated clothing choices had the lowest risk of mental health problems at follow-up, with the highest risk being in those with marginalised clothing choices. These findings were sustained even after adjustment for place of birth, religious group and number of years resident in the United Kingdom.

Table 2 Risk of being a strengths and difficulties questionnaire case in the second phase, in relation to cultural identity in the first phase, stratified by ethnic group

\begin{tabular}{|c|c|c|c|c|c|c|c|c|}
\hline \multirow[b]{2}{*}{ Ethnic group } & \multirow[b]{2}{*}{ Cultural identity } & & \multicolumn{3}{|l|}{ Unadjusted } & \multicolumn{3}{|l|}{ Adjusted* } \\
\hline & & & OR (95\% CI) & p Value & $\mathbf{N}, \mathbf{R}^{2}$ & OR (95\% Cl) & $\mathrm{p}$ Value & $\mathbf{N}, \mathbf{R}^{2}$ \\
\hline \multicolumn{9}{|l|}{ Model 1} \\
\hline \multirow{3}{*}{ White British } & & Traditional & $1.1(0.5$ to 2.4$)$ & 0.83 & & 1.0 (0.4 to 2.5$)$ & 0.94 & \\
\hline & & Assimilated & $1.1(0.6$ to 1.9$)$ & 0.71 & $\mathrm{~N}=351$ & 1.1 (0.4 to 2.7$)$ & 0.89 & $N=326$ \\
\hline & & Marginalised & $0.7(0.3$ to 1.7$)$ & 0.44 & & $0.7(0.2$ to 1.9$)$ & 0.44 & \\
\hline \multirow[t]{4}{*}{ Bangladeshi } & Friendship choice & Integrated & 1 & & & & & \\
\hline & & Traditional & $0.8(0.4$ to 1.5$)$ & 0.47 & & 0.9 (0.5 to 1.7$)$ & 0.77 & \\
\hline & & Assimilated & $1.9(0.6$ to 6.0$)$ & 0.31 & $N=453$ & $1.6(0.5$ to 5.6$)$ & 0.43 & $N=413$ \\
\hline & & Marginalised & $1.2(0.5$ to 2.6$)$ & 0.71 & & 1.3 (0.5 to 3.8 ) & 0.58 & \\
\hline \multicolumn{9}{|l|}{ Model 3} \\
\hline \multicolumn{9}{|l|}{ Model 4} \\
\hline \multirow[t]{4}{*}{ Bangladeshi } & Clothing choices & Integrated & 1 & & & 1 & & \\
\hline & & Traditional & $0.3(0.1$ to 0.9$)$ & 0.02 & & $0.17(0.06$ to 0.5$)$ & 0.007 & \\
\hline & & Assimilated & $1.3(0.8$ to 2.3$)$ & 0.26 & $N=454$ & $1.0(0.5$ to 1.9$)$ & 0.67 & $\mathrm{~N}=412$ \\
\hline & & Marginalised & 0.93 (0.5 to 1.8$)$ & 0.84 & & $0.82(0.4$ to 1.6$)$ & 0.12 & \\
\hline
\end{tabular}

\footnotetext{
OR, Odds ratio.
}

${ }^{*}$ Adjustment for age (continuous), gender and year group interaction, eligibility for free school meals, car ownership, number of rooms in the house, religion, place of birth, phase 1 strengths and difficulties questionnaire score as a continuous variable, social support from friends score as a continuous variable. All analyses weighted for proportion of nonresponders per cluster (using pw in Stata) and standard errors adjusted for cluster sampling using cluster in Stata. 
Table 3 Risk of phase 2 mental health problems by cultural identity based on clothing choices at phase 1, stratified by gender and ethnic group

\begin{tabular}{|c|c|c|c|c|c|c|c|c|}
\hline & \multirow[b]{2}{*}{ Cultural identity } & & \multicolumn{3}{|l|}{ Unadjusted } & \multicolumn{3}{|l|}{ Adjusted* } \\
\hline & & & OR $(95 \%$ Cl) & p Value & $N_{R^{2}}$ & OR $(95 \% \mathrm{Cl})$ & p Value & $\mathrm{N} \mathrm{R}^{2}$ \\
\hline White British & Boys & Integrated & 1 & & 174 & 1 & & 150 \\
\hline \multirow[t]{5}{*}{ Clothing choice } & & Traditional & $1.3(0.5$ to 3.7$)$ & 0.57 & 3.2 & $1.4(0.5$ to 4.1$)$ & 0.53 & 7.1 \\
\hline & & Assimilated & 3.1 (0.4 to 22.8 ) & 0.27 & & $2.4(0.4$ to 13.5$)$ & 0.32 & \\
\hline & & Marginalised & 3 (1.2 to 7.7$)$ & 0.03 & & 2.9 (1.1 to 7.9$)$ & 0.04 & \\
\hline & Girls & Integrated & 1 & & 177 & 1 & & 167 \\
\hline & & Marginalised & 3.9 (0.98 to 15.5$)$ & 0.05 & & $4(0.9$ to 18.5$)$ & 0.08 & \\
\hline Bangladeshi & Boys & Integrated & 1 & & 239 & 1 & & 213 \\
\hline \multirow[t]{4}{*}{ Clothing choice } & & Traditional & $0.6(0.1$ to 2.5$)$ & 0.45 & 3.1 & $0.4(0.1$ to 1.8$)$ & 0.24 & 9.4 \\
\hline & & Assimilated & $0.3(0.08$ to 1$)$ & 0.05 & & $0.3(0.1$ to 1$)$ & 0.04 & \\
\hline & & Marginalised & $1.3(0.5$ to 3.5$)$ & 0.59 & & $1.2(0.4$ to 3.8$)$ & 0.80 & \\
\hline & Girls & Integrated & 1 & & 215 & 1 & & 198 \\
\hline
\end{tabular}

OR, Odds ratio.

${ }^{*}$ Adjustment for baseline characteristics: age, year group, eligibility for free school meals, car ownership, number of rooms in house, and mental health problems at baseline (strengths and difficulties questionnaire case or not).

\section{School-level effects}

There was no significant school-level variation in mental disorder for Bangladeshi pupils (beta $<0.001$, standard error $<0.001)$ but there was some school-level variance of mental disorder for white British pupils (beta 0.25 , standard error 0.26). There were no significant school-level effects of traditional identity on the risk of individual-level mental health problems. Individual-level effects of cultural identity were sustained for white British pupils but not for Bangladeshi pupils, probably as these analyses were not weighted.

\section{DISCUSSION}

Clothing preferences appear to influence mental health prospectively, with different effects apparent by gender. Bangladeshi girls were most likely to benefit from traditional clothing choices, and white British girls benefited from integrated clothing choices. School-level effects were not apparent.

\section{Methodological limitations}

Clothing choices and mental health are complex variables determined by a number of interrelated factors including financial strain, cultural beliefs, gender roles, immediate social networks, and the impact of the cultural identity of parents and peers; clothing choice may also be influenced by fashion trends and needs better investigation. ${ }^{38} 394142$ We adjusted for several indicators of socioeconomic status, but the findings may still reflect residual confounding as a result of poverty. This may restrict spending on clothes, or more conservative parents may restrict experimentation with clothes and this may lead to distress. Furthermore, in multicultural environments, where pupils endorsed a preference for other cultures' clothes or friends, this may involve identifications with many other groups rather than only one other group. Nonetheless, the most important determinant of risk may be the degree of identification with "other" cultures and alienation from ones own culture, irrespective of the specific cultures that constitute the "other". Larger samples are needed to undertake stratified analyses by gender and by ethnic group and by other characteristics that define a cultural group. We did not have an independent measure of cultural identity in schools and so had to aggregate identities of pupils. Independent measures are not routinely available.

\section{Ethnic and gender effects}

The absence of associations between friendship-based measures of identity and mental disorder contradict previous crosssectional studies; ${ }^{67}$ the cross-sectional findings may be explained if those with mental health problems were less able to make friends from other cultural groups. These findings also indicate that when the effects of cultural identity are compared across ethnic groups using the same questions, cultural identity items may actually show effects in different directions by ethnic group and within ethnic groups. Therefore, identity experiences should not be reduced to a single score or measure of overall identity.

Clothing choices as measures of cultural identity are important for girls and boys, but the pattern of associations differs across ethnic groups. A preference for traditional clothing among Bangladeshi girls appears to confer a lower risk of mental disorder, perhaps mediated through a restricted or traditional upbringing that is protective for mental health for girls in early teenage years. This traditional upbringing may include greater religious adherence and perhaps less exposure to unfamiliar and therefore culturally challenging life events.

In contrast to Bangladeshi girls, white British girls with traditional clothing preferences had a higher risk, and those with integrated clothing preferences had the lowest risk of mental disorder. White British girls who preferred clothes from other cultural groups (assimilated) have an even higher risk of mental heath problems than white British girls choosing traditional clothing. Girls from both cultures may be encouraged to be more conservative than boys and to conform to cultural practices of their own cultural group, ${ }^{39}$ but they are also expected to conform to a wider dress code within local peer groups and schools and neighbourhoods. For white British girls, the complete adoption of another culture's clothing preferences may generate conflict with families and peers, especially if this 


\section{What is already known on this subject}

- Migration and cultural change in lifestyle are common in urban and industrialised societies around the world

- Strategies to ensure harmonious relationships between diverse ethnic groups now make comment on whether certain cultural practices should be discouraged or banned in public spaces, but the mental health impact of social policies are not commonly reported

- Acculturation and cultural identity are known to show associations with mental health status among young people

\section{What this study adds}

- This is the first prospective study investigating cultural identity and mental health status among young people living in a culturally diverse society

- Clothing preferences as markers of cultural identity influence the risk of later mental health problems

- In particular, Bangladeshi girls who prefer traditional clothing and white British girls who choose integrated clothing have the lowest risk of later mental health problems

is an expression of emerging divergence in identity between girls and their peers and parents.

Bangladeshi boys may be more motivated to and permitted to wear western clothing because of fewer religious and cultural constraints when compared with Bangladeshi girls. Bangladeshi boys who entirely prefer western clothing have a lower risk of mental health problems; this may allow them to "fit in" with non-Bangladeshi social groups. If this makes them less conspicuously different, they may not attract discrimination while securing friendships; alternatively, clothing preferences here may be a proxy for acquiring knowledge about how to develop and sustain mutually advantageous relationships with better social support. Friendship-based measures of identity did not, however, confirm this and our findings on clothing were sustained even after adjustment for social support from friends. One explanation is that identity as expressed in clothing choice may improve self-esteem, which may buffer against distress and conflicts as a resiliency factor. Traditional clothing choices may bolster self-esteem through a positive group identity; this may help with psychological adaptation if these choices reflect the local communities' "lay policy" for managing cultural adaptation when national policies are ineffective. ${ }^{43}$

There may be different cultural reasons for clothing selection and constraints on selection by gender. A study of white and black American adolescents reported that boys used clothes to achieve their aspired and ideal identity as they were dealing with autonomy issues while separating from parents; whereas girls used clothes to enhance happiness, at a time when they are primarily dealing with separation from the mother. ${ }^{27}$ In support of this, a study of East Indian-Caribbean adolescents reported that male adolescents were more likely to work outside of the home at a younger age, and were less likely to face social controls compared with girls. ${ }^{42}$ Girls were more likely to wear Indian clothing to weddings and religious services, whereas boys distanced themselves from their Indian origins. Indeed, the significance attached to clothing as a marker of group behaviour, identity, and individual identity can not be assumed

\section{Policy implications}

- Culturally determined choice in clothing is strongly associated with later mental disorders among Bangladeshi and white British young people, and the effects also vary by gender

- The findings inform health policy and policies affecting educational and social institutions caring for young people in multicultural societies

- Policies and practices that encourage young people to move from traditional forms of identity to integrated ones require further development, research and evaluation

to be similar across cultural groups. The hijab, for example, has come to represent a particular commitment to religious identity and practice, beyond simply being a form of clothing. If we had asked specific questions on the hijab of white British people, the question would not make as much sense. Therefore, our approach of asking about clothing traditional to or distinct from the culture of the respondent overcomes this to some extent. Furthermore, the terms "traditional clothing identity" and "integrated clothing identity" are clearly defined in distinct ways for each ethnic group, therefore interpretation of the findings should not assume equivalence of social or political investment in clothing choices, nor that the impact of acculturation is only evident for the minority in-migrating group. In our setting, Bangladeshi people can represent the dominant ethnic grouping; our study also shows the impact of cultural identity on the national majority population when they are locally a minority.

\section{Future research and policy}

Gender roles may be the most important determinants of conformity to cultural norms of dress; ${ }^{67}$ our findings may be explained if gender roles that are unique to any one culture exert a more sustained influence over time during vulnerable periods in adolescence. ${ }^{2} 3244$ Conformity to gender roles may avoid cultural conflicts with parents and with peers; by delaying maturation and experimentation, the onset of intimate and mixed gender relationships may be delayed. ${ }^{123038} 39$ The relationship of gender roles and clothing as an expression of identity may be a fruitful area for future studies, ${ }^{27}{ }^{40}$ especially in diverse cultural groups with distinct norms of gender roles. Studies such as the Immigrant Youth in Cultural Transition study can help unravel the influence of policies and practices on healthy cultural adaptation of young people..$^{45}$ Future studies need to take a prospective design, consider the impacts on the host as well as the immigrant population, and must be cognisant of local neighbourhood effects and local "lay policies" alongside national policies to manage acculturation.

Acknowledgements: The study was commissioned by East London and The City Health Authority to inform their Health Action Zone and the authors would like to thank them for their support. They also thank the Department of Health (UK), and Tower Hamlets, City and Hackney and Newham Primary Care Trusts for additional funding. The authors are grateful for the support of the schools, parents and students involved in this study.

Funding: Additional funding was supplied by the UK Department of Health and Tower Hamlets, City and Hackney and Newham Primary Care Trusts.

Competing interests: None.

Contributors: KB, SS, RV, JH, CC were all involved in the original and follow-up phases of the RELACHS study, including design, conception and data analyses of both phases of data. KB specifically designed and managed the data on cultural identity, and analyses related to hypotheses on cultural identity and conceived the idea for this 
paper. JH is the study statistician. These authors have contributed to the design of the data analyses and editing of consecutive version of the paper. YK and EK are PhD students who have contributed to data analyses, the design of the analyses and the RELACHS study and have commented on consecutive drafts of the paper. KB is the guarantor.

\section{REFERENCES}

1. Hankin BL, Abramson LY. Development of gender differences in depression: description and possible explanations. Ann Med 1999;31:372-9.

2. Ge X, Conger RD, Elder GH Jr. Pubertal transition, stressful life events, and the emergence of gender differences in adolescent depressive symptoms. Dev Psychol 2001;37:404-17.

3. Graber JA, Lewinsohn PM, Seeley JR, et al. Is psychopathology associated with the timing of pubertal development? J Am Acad Child Adolesc Psychiatry 1997;36:176876.

4. Roberts RE, Attkisson CC, Rosenblatt A. Prevalence of psychopathology among children and adolescents. Am J Psychiatry 1998;155:715-25.

5. Berry J. Immigration, acculturation, adaptation. Appl Psychol: an international review 1997;46:5-34.

6. Bhui K, Lawrence A, Klineberg E, et al. Acculturation and health status among African-Caribbean, Bangladeshi and White British adolescents. Validation and findings from the RELACHS study. Soc Psychiatry Psychiatr Epidemiol 2005;40:259-66.

7. Bhui K, Stansfeld S, Head J, et al. Cultural identity, acculturation, and mental health among adolescents in east London's multiethnic community. J Epidemiol Community Health 2005:59:296-302.

8. Phinney JS. Ethnic identity in adolescents and adults: review of research. Psychol Bull 1990;108:499-514.

9. Minister calls for veil-wearing teaching assistant to be sacked. Independent online. http://news.independent.co.uk/uk/politics/article1876677.ece laccessed 17 Jan 2008).

10. John Lichfield: So were the French right all along? Independent online http://comment.independent.co.uk/commentators/article1902146.ece) (accessed 17 Jan 2008).

11. Tajfel H. Social identity and intergroup relations. Cambridge: Cambridge University Press, 1982

12. Yancey AK, Siegel JM, McDaniel KL. Role models, ethnic identity, and health-risk behaviors in urban adolescents. Arch Pediatr Adolesc Med 2002;156:55-61.

13. Root MP. Back to the drawing board: methodological issues in research on multiracial people. In: MP Root, ed. Racially mixed people in America. London: Sage Publications, 1992:181-189.

14. Redfield R, Linton R, Herskovits MJ. Memorandum on the study of acculturation. Am Anthropol 1936;38:149-52.

15. Fullilove MT. Psychiatric implications of displacement: contributions from the psychology of place. Am J Psychiatry 1996;153:1516-23.

16. Neeleman J, Wilson-Jones C, Wessely S. Ethnic density and deliberate self harm; a small area study in south east London. J Epidemiol Community Health 2001;55:8590.

17. Boydell J, van Os J, McKenzie K, et al. Incidence of schizophrenia in ethnic minorities in London: ecological study into interactions with environment. BMJ 2001;323:1336-8.

18. Cederblad M, Hook B, Irhammar M, et al. Mental health in international adoptees as teenagers and young adults. An epidemiological study. J Child Psychol Psychiatry 1999;40:1239-48.

19. Fichter MM, Elton M, Diallina M, et al. Mental illness in Greek and Turkish adolescents. Eur Arch Psychiatry Neurol Sci 1988;237:125-34

20. Davies LC, McKelvey RS. Emotional and behavioral problems and competencies among immigrant and non-immigrant adolescents. Aust NZ J Psychiatry 1998:32:658-65.
21. Berry W. Acculturation as varieties of adaptation. In: Padilla A, ed. Acculturation: theory, models and some new findings. Bolder, Co: Westview, 1980:9-25.

22. Bhugra D, Bhui K. The Asian Cultural Identity Schedule: an investigation of culture and deliberate self harm. Int J Methods Psychiatr Res 2000;8:212-18.

23. Graber JA, Brooks-Gunn J, Warren MP. The antecedents of menarcheal age: heredity, family environment, and stressful life events. Child Dev 1995;66:346-59.

24. Claes ME. Friendship and personal adjustment during adolescence. J Adolesc 1992;15:39-55

25. Clark ML, Ayers M. Friendship similarity during early adolescence: gender and racial patterns. J Psychol 1992;126:393-405.

26. Horenczyk G, Tatar M. Friendship expectations among immigrant adolescents and their host peers. J Adolesc 1998;21:69-82.

27. Hamm JV. Do birds of a feather flock together? The variable bases for African American, Asian American, and European American adolescents' selection of similar friends. Dev Psychol 2000:36:209-19.

28. Fotheringham MJ, Sawyer MG. Do adolescents know where to find help for mental health problems. A brief report. J Paediatr Child Health 1995;31:41-3.

29. Dubois DL, Hirsch BJ. School and neighborhood friendship patterns of blacks and whites in early adolescence. Child Dev 1990;61:524-36.

30. Sontag S. Clothing in the self system of adolescents: relationships among values, proximity of clothing to self, clothing interest, anticipated outcomes, and perceived quality of life. Michigan State University Research Report 556098, 1997. http:// web1.msue.msu.edu/msue/imp/modrr/rr556098.html (accessed 17 Jan 2008).

31. Sweeney MM, Zionts P. The "second skin": perceptions of disturbed and nondisturbed early adolescents on clothing, self-concept, and body image. Adolescence 1989:24:411-20.

32. Stansfeld SA, Haines MM, Head JA, et al. Ethnicity, social deprivation and psychological distress in adolescents: school-based epidemiological study in east London. Br J Psychiatry 2004;185:233-8.

33. Goodman R, Meltzer H, Bailey V. The Strengths and Difficulties Questionnaire: a pilot study on the validity of the self-report version. Int Rev Psychiatry 2003;15:173-7.

34. Goodman R, Ford T, Simmons $\mathrm{H}$, et al. Using the Strengths and Difficulties Questionnaire (SDO) to screen for child psychiatric disorders in a community sample. Br J Psychiatry 2000;177:534-9.

35. Goodman R. The Strengths and Difficulties Questionnaire: a research note. J Child Psychol Psychiatry 1997;38:581-6.

36. Meltzer H, Gatward R, Goodman R. et al. Mental health of children and adolescents in Great Britain. London: The Stationery Office, 2000.

37. Prescott-Clarke P, Primatesta P. Health Survey for England. The health of young people 1995-97. London: The Stationery Office, 1998.

38. Ellis-Schwabe M, Thornburg HD. Conflict areas between parents and their adolescents. J Psychol 1986;120:59-68.

39. Ben-Shalom U, Horenczyk G. Acculturation orientations. J Cross Cult Psychol 2003:34:176-88.

40. Zimet GD, Dahlem NW, Zimet SG, et al. The multidimensional scale of social support. J Pers Assess 1988;52:30-41.

41. Cooksey EC, Mott FL, Neubauer SA. Friendships and early relationships: links to sexual initiation among American adolescents born to young mothers. Perspect Sex Reprod Health 2002;34:118-26.

42. Warikoo N. Gender and ethnic identity among second generation Indo-Caribbeans. Ethn Racial Stud 2005:28:803-31.

43. Vedder $\mathbf{P}$, van de Vijver FJR, Liebkind K. Predicting immigrant youths' adaptation across countries and ethnocultural groups. In: Berry J, Phinney JS, Sam DL, Vedder P, eds. Immigrant youth in cultural transition. Mahwah, New Jersey: Lawrence Erlbaum Associates, 2006:143-166

44. Sweeney MM, Zionts P. The "second skin": perceptions of disturbed and nondisturbed early adolescents on clothing, self-concept, and body image. Adolescence 1989;24:411-20.

45. Berry J, Phinney JS, Sam DL, et al. Immigrant youth in cultural transition. Mahwah, New Jersey: Lawrence Erlbaum Associates, 2006. 